\title{
Strates
}

STRATES Matériaux pour la recherche en sciences sociales

Hors-série | 2002

Parcours dans la recherche urbaine, Michel Rochefort, un géographe engagé

\section{Une forte implication dans les institutions de développement}

Michel Rochefort et l'Iedes

Maxime Haubert et Catherine Paix

\section{(2) OpenEdition \\ Journals}

\section{Édition électronique}

URL : http://journals.openedition.org/strates/569

DOI : $10.4000 /$ strates.569

ISSN : $1777-5442$

\section{Éditeur}

Laboratoire Ladyss

Édition imprimée

Date de publication : 1 janvier 2002

ISSN : 0768-8067

\section{Référence électronique}

Maxime Haubert et Catherine Paix, « Une forte implication dans les institutions de développement », Strates [En ligne], Hors-série | 2002, mis en ligne le 18 mai 2005, consulté le 08 septembre 2020. URL : http://journals.openedition.org/strates/569; DOI : https://doi.org/10.4000/strates.569

Ce document a été généré automatiquement le 8 septembre 2020

Tous droits réservés 


\title{
Une forte implication dans les institutions de développement
}

Michel Rochefort et l'Iedes ${ }^{1}$

\author{
Maxime Haubert et Catherine Paix
}

Une préoccupation majeure : le Tiers-Monde

1 Depuis le début des années soixante-dix, tu t'es beaucoup investi à l'Institut du développement économique et social, que ce soit au niveau de l'enseignement, de la recherche, ou des publications; peux-tu rappeler dans quelles circonstances tu es entré à l'Iedes, et ce qui t'a conduit à t'y intéresser?

2 Cela correspond à mon tournant Tiers-Monde au tout début des années soixante-dix. J'avais déjà une connaissance des problèmes du développement, mais jusque-là je n'avais pas beaucoup utilisé mes connaissances. Après mon grand voyage en 1956, qui m'avait mené de New-York au Brésil, où je suis resté sept mois pour faire ma thèse complémentaire, et où j'ai découvert - grâce en partie à l'aviation militaire - presque toutes les régions du Brésil, - l'Amazonie, le Sud-Ouest, le Nord-Est etc. - j'ai repris mes activités françaises. Et j'en ai fait autant à la suite de mon séjour au Brésil en 1960/61, durant lequel j'avais abordé un certain nombre de problèmes du développement au travers de mon enseignement et de ma direction de recherche au Conseil National de Géographie $^{2}$. C'est à cette époque que je me suis surtout lancé dans les métropoles d'équilibre. J'étais invité un peu partout pour faire des conférences sur ce sujet, et à l'université j'étais au jury d'agrégation. Ce n'était donc pas le moment pour moi de penser au Tiers-Monde. Et ce n'est que par la suite, lorsque j'ai vu que l'aménagement du territoire en France se fermait pour moi, que j'ai été amené à véritablement m'investir sur le Tiers-Monde, - les deux choses étant là curieusement liées. J'ai d'abord été appelé par les économistes de l'Orstom en 1967. J. Perrin, le spécialiste des problèmes d'économie régionale de Côte d'Ivoire, puis A. Nicolaï, professeur d'économie à Nanterre, qui ont été successivement président du Comité technique d'Économie/Démographie de l'Orstom, m'ont demandé d'en faire partie en tant que géographe. Ils m'ont très gentiment fait visiter l'Afrique au cours d'une tournée Orstom, pour que je puisse avoir un contact avec Dakar et Abidjan. Et finalement, en 
1971, le directeur général de l'Orstom m'a demandé si j'accepterais de présider le Comité technique de la section Économie/Démographie dans la mesure où j'étais un observateur plus ou moins averti des problèmes du Tiers-Monde et où j'étais extérieur à l'organisme. J'ai été président de cette section pendant dix ans, jusqu'en 1981. À l'époque, j'avais donc une connaissance des problèmes du Tiers-Monde à la fois par ma connaissance brésilienne, par mon insertion dans l'Orstom qui m'avait fait découvrir l'Afrique sub-saharienne, et par le grand voyage d'étude que j'avais fait à l'occasion d'un Congrès à New Delhi qui m'a fait découvrir l'Inde : j'avais en tête la question des convergences et divergences au sein des pays du Tiers-Monde. Mais je ne connaissais l'Iedes que de nom, et dans mes cours, j'abordais la question du Tiers-Monde sans que cela soit vraiment très structuré. En 1970, Milton Santos avait créé à l'Iedes une équipe de recherche " Analyse régionale et aménagement de l'espace ", et c'est peu après qu'il ait quitté l'Iedes que j'y suis rentré. J'ai alors repris la direction de cette équipe, - à la demande de Catherine Paix qui était aussi dans l'équipe du laboratoire ${ }^{3}$, avec Hélène Lamicq, Claire Biétry et Marie-Claude Guerrini - et la responsabilité de l'option d'enseignement et du diplôme. C'était en 1973-1974, à peu près au même moment où j'ai commencé à donner des cours sur le Tiers-Monde à l'Institut de géographie. Et jusqu'en 1980, je me suis investi, d'une part, dans les recherches de cette équipe, et notamment dans ce que Charles Morazé a appelé le «Livre rouge ${ }^{4}$ » de l'Iedes, qui était un essai de classification des pays du Tiers-Monde, et d'autre part, au niveau du diplôme " Analyse régionale et aménagement de l'espace » auquel j'ai associé Patrick Castex.

3 Le diplôme a ensuite disparu dans le cadre d'une réorganisation de l'Iedes et j'ai pris d'autres responsabilités. En 1981, j'ai quitté la présidence du Comité de l'Orstom parce que tous les Comités techniques ont été supprimés. Mais j'ai présidé la Commission de financement de la recherche urbaine dans les PVD au ministère de la Recherche, ce qui a représenté un gros travail que j'ai mené pendant six ans, et je ne suis revenu à l'Iedes que lorsque Maxime Haubert en est devenu le directeur et que l'on a parlé ensemble de lancer de nouveaux diplômes. J'avais pour ma part l'idée de créer un diplôme d'aménagement urbain, qui a effectivement existé, et qui vient d'être supprimé.

L'Iedes : un brassage d'idées

$4 \quad$ Qu'est ce qui t'a attaché à cet Institut ?

5 Quand je suis entré à l'Iedes, c'était vraiment dans l'optique d'après 1968. J'avais ma propre culture du Tiers-Monde, forgée par cette triple expérience directe de l'Amérique latine, de l'Afrique, et de l'Inde, mais aussi du Maghreb (à partir de 1965-67, j'ai dirigé des thèses en Tunisie, au Maroc), et étayée par les lectures de l'époque, Samir Amin, Gunder Frank, Pierre Jallée, etc., et par les travaux de l'équipe du laboratoire qui m'incitait à travailler sur l'impérialisme. L'Iedes était donc un cadre qui correspondait à ce que j'attendais du point de vue idéologique - dans la mesure où pour moi il n'y a pas de sciences humaines sans idéologie. J'y ai trouvé une correspondance de point de vue et de valeurs que je ne trouvais pas à l'université de Paris I, où mes collègues de l'UFR 02 de l'époque faisaient de l'économie du développement qui ne répondait en rien à ce que moi je ressentais des vrais problèmes socio-économiques du Tiers-Monde. À ce moment-là, je m'y intéressais presque davantage qu'aux problèmes géographiques. L'organisation de l'espace de ces sociétés et ses différences ne m'intéressaient qu'en tant qu'expression dans le territoire de la spécificité des structures socio-économiques et formes de domination. Je me situais donc tout à fait dans l'optique transdisciplinaire 
de l'Iedes, contrairement aux «officiels» de l'économie du développement qui n'avaient pas de contact avec la réalité profonde des sociétés du Tiers-Monde, et cela m'a paru être une Maison où je pouvais m'exprimer. C'est ce que j'ai essayé de faire, que ce soit en dirigeant des travaux, en reprenant le diplôme ou en collaborant au développement de l'Institut.

6 Après quoi j'ai fait des tas d'autres choses y compris sur le Tiers-Monde - en particulier au ministère de la Recherche et dans mes propres recherches avec le groupe qui travaillait sur le secteur informel, Henri Coing, Hélène Lamicq, Philippe Hugon, etc. Puis quand Maxime Haubert est devenu directeur, j'ai retrouvé l'envie d'être dans cette Maison, parce qu'elle avait été menacée et que je sentais que c'était les forces réactionnaires qui l'attaquaient. Je me suis donc réinvesti en tant qu'enseignant, et en essayant d'aider l'Iedes à fonctionner, ce qui a partiellement réussi pendant un temps.

Est-ce qu'il y a des personnalités qui t'ont marqué à l'Iedes?

8 Oui, des personnalités qui avaient parfois des positions différentes des miennes, mais qui m'ont amené à préciser mes idées. Il y avait Patrick Castex, qui était un marxiste vraiment épuisant avec son modèle prussien, il y avait Moïse Ikonicoff, avec son capitalisme périphérique etc., tous des gens qui finalement se situaient dans la perspective des grands, Samir Amin, Gunder Frank, etc., et j'aimais bien discuter avec eux. En dehors de l'Iedes, j'ai aussi discuté avec Celso Furtado, quand il était professeur à Paris I, avec Alain Touraine. Mais après, il y a eu surtout Henri Coing, qui avait quitté l'Iedes quand j'y étais, et Philippe Hugon, qui m'ont beaucoup appris. Tous ont été des interlocuteurs tout à fait importants pour moi parce que, dans mon approche du territoire, il y avait la nécessité de comprendre les mécanismes économiques et sociologiques. L'Iedes, c'était un brassage d'idées que j'aimais bien.

Et avec Milton Santos?

10 Milton n'était plus à l'Iedes à ce moment-là, mais j'ai beaucoup appris de lui au moment où il a écrit ses premiers livres, notamment L'espace partagé $e^{5}$. Il ne faut pas oublier qu'il a été l'un des pionniers du secteur informel avec les travaux qu'il a dirigé à l'Iedes sur Tlemcen et Saïda en Algérie. J'ai beaucoup discuté avec lui avant l'Iedes et, dans mon approche du Tiers-Monde, c'est quelqu'un qui a compté.

11 La vie était toujours très agitée, très conflictuelle à l'Iedes; quels souvenirs en as-tu?

12 Certains disent que je suis un diplomate. Dans les conflits, j’ai en effet toujours écouté les uns et les autres en évitant au maximum de m'investir dans le conflit ouvert;à l'Iedes j'ai eu la même politique, j'ai écouté tout le monde. Il y avait des gens que j'aimais et d'autres que je n'aimais pas, parce que je suis assez sélectif dans mes affections. Mais j'ai bien écouté et, par derrière éventuellement, j'ai œuvré à aider ceux que j'estimais.

Au cœur des débats : domination et diversité du Tiers-Monde

13 Dans les années soixante-dix, tu dirigeais l'équipe de recherche "Analyse régionale», dont les préoccupations n'étaient pas très éloignées de celles de l'équipe du Laboratoire de Géographie humaine, on avait des réunions toutes les semaines, on discutait beaucoup. Qu'est ce que tu as retenu de ces recherches?

14 À ce moment-là je m'investissais complètement, aussi bien au Laboratoire de Géographie humaine qu'à l'Iedes, et je pense que cette équipe m'a beaucoup ouvert les yeux sur les critères de différences au sein des pays du Tiers-Monde. Le Tiers-Monde et la domination, on était tous d'accord, vous étiez des tiers-mondistes convaincus. Mais 
vous aviez une approche qui était intéressante, parce que, en fonction de cette problématique de la domination, vous cherchiez des indicateurs susceptibles d'expliquer la diversité du Tiers-Monde, et à prouver de façon quantifiée leur validité. C'est ce qui m'a intéressé dans ce fameux « Livre Rouge ».

15 Si l'on se resitue à cette époque, en quoi, selon toi, cette approche de la domination et de ses implications sur l'organisation de l'espace dans les pays du Tiers-Monde, était-elle novatrice en géographie?

Il n'y avait déjà pas beaucoup de gens qui en parlaient, à part Yves Lacoste ${ }^{6}$. Par conséquent, c'était novateur. Mais vous aviez aussi une approche plus précise, même si vous n'aviez pas une très grande connaissance du Tiers-Monde. À ce moment-là, c'était en 1973/74, on a vraiment creusé une approche, qui, partant d'indicateurs liés à une doctrine unitaire du Tiers-Monde, a consisté à rechercher comment classer des catégories de pays du Tiers-Monde, comment expliquer leur diversité. Cela a été une période de mûrissement dans la réflexion de cette question d'unité/diversité du TiersMonde, avec comme idée centrale le fait qu'en tout état de cause il y a une spécificité de ces pays par rapport au monde développé parce que ce sont des espaces qui ont subi la domination.

17 Et pour ce qui est de la géographie, il faut se replacer à l'époque. Il y avait des géographes qui connaissaient le Tiers-Monde mais qui n'avaient aucune idéologie. C'était le cas de ceux de l'école géographique dite française du Brésil, qui avaient une approche de terrain dénuée d'idéologie. Et puis il y avait les géographes qui approchaient la réalité du Tiers-Monde, en se refusant de l'appeler Tiers-Monde, c'était la géographie tropicale. Le Ceget ${ }^{7}$, par exemple, qui était dirigé par des tropicalistes, a refusé dans les années soixante-dix une allocation de recherche pour une thèse sur le Chili, parce que le Chili était en dehors des tropiques! En ce qui me concerne, j'avais une idéologie et une expérience de terrain. Et c'est sur cette base que j'ai commencé à faire des cours généraux sur la question de l'unité et de la diversité du Tiers-Monde. Cela a toujours été l'essence de ce que j'ai essayé de faire, que ce soit en dirigeant des recherches ou dans mon enseignement, et je pense que la géographie avait besoin de cette approche du Tiers-Monde.

18 Si débat il y avait, est-ce que tu te souviens des discussions que tu as pu avoir à l'époque avec les géographes autour de ces questions-là?

19 Il n'y en avait pas beaucoup, car la géographie était très clivée. On discutait entre nous, ceux qui se croyaient marxistes ou qui l'étaient, mais avec les autres on discutait peu. Ce que faisait $P$. Gourou, le grand-père de la géographie tropicale, ne m'intéressait pas vraiment. Par contre, avec Gilles Sautter, qui était un ami, j'ai discuté. Mais d'une façon générale j'ai eu peu d'autres grandes discussions, et les accrochages n'avaient lieu que lorsqu'il y avait un équilibre des forces. Cela a été le cas, par exemple, au colloque de Yaoundé en 1973 sur les problèmes urbains, avec Perrin, Gilles Sautter, Paul Pelissier et d'autres chercheurs de l'Orstom. Je me souviens qu'après qu'ils aient parlé, pour dire en gros que l'Afrique se portait bien - ils faisaient référence au miracle ivoirien auquel ils croyaient -, j'ai fait une intervention, en montrant que tant que l'économie resterait une économie extravertie et dominée, il y aurait toujours les mêmes problèmes. Gilles Sautter a alors repris la parole en disant que ma démonstration était très forte, mais que, si on en restait là, cela voulait-il dire que l'on ne pouvait rien faire? Il essayait de se défendre en disant que j'étais un pessimiste désespéré mais il n'a pas pu casser mon raisonnement. Après, Perrin s'est également opposé à moi, en soutenant qu'il y avait 
des modèles de développement régional, ce que les chercheurs de l'époque qui travaillaient sur Bouaké ont démenti en appuyant ma position, à savoir qu'il n'y avait pas de modèle de développement régional à partir de Bouaké. Cela a été une belle discussion. Mais en général, il y avait surtout deux écoles qui s'opposaient et qui ne s'écoutaient pas, plutôt que de vraies discussions.

A l'Iedes : un contact essentiel avec le Tiers-Monde ; un public motivé et enrichissant

Au niveau de l'enseignement, tu as beaucoup apporté à l'Iedes, et tu n'as jamais été le genre de prof qui se contente de donner ses deux heures de cours. Qu'est ce que l'Iedes t'a apporté?

D'abord un public. Le contact avec les étudiants, avec ceux qui vous écoutent, que ce soit des chercheurs ou autres, c'est toujours important. J'ai beaucoup appris, en particulier des exposés qui étaient faits dans le cadre de mon diplôme «Problèmes et Pratiques de l'aménagement urbain dans les PVD », qui attirait des gens motivés, parce qu'ils venaient là avec l'idée qu'il fallait qu'ils retournent mieux formés dans leur pays, et pour y faire quelque chose. Leur problème, c'était de savoir quoi faire pour que les villes de leur pays marchent mieux ou moins mal, et de ce fait, même s'ils étaient parfois trop utopistes ou idéalistes, ou au contraire trop pessimistes, les discussions qui suivaient leurs exposés m'apprenaient beaucoup, d'autant qu'en dehors du Brésil, je n'avais pas tellement de contacts directs avec les gens d'autres pays. L'Iedes était donc pour moi un lieu privilégié de rencontres. Dakar, Abidjan, Yaoundé, j'y étais allé au titre de l'Orstom entre 1970 et 1981, mais c'était un peu dans le milieu fermé de l'Orstom, et avec des chercheurs qui ne partageaient pas forcément mes idées. Mon contact direct avec le Tiers-Monde, l'Afrique en particulier, s'est donc fait souvent par le biais des étudiants qui venaient de ces pays. Ils avaient une demande, que je ne pouvais pas satisfaire bien entendu, parce que ce n'est pas moi qui pouvais apporter des solutions aux problèmes urbains du Tiers-Monde, mais c'était extrêmement enrichissant.

Pendant toutes les années où j'ai enseigné à l'Iedes je n'ai jamais fait le même cours. À chaque fois je changeais, soit dans les exemples que je prenais, soit dans la façon d'aborder les problèmes, et cela, grâce aux apports des étudiants de l'année précédente avec lesquels j'avais discuté. Cela a été le cas en particulier pour le secteur informel. J'ai beaucoup reçu des interventions, parfois très critiques, même un peu ulcérées, des étudiants africains, qui défendaient l'idée que le secteur informel était une forme traditionnelle de production issue de l'artisanat qui était propre à leur société, et qu'il ne fallait pas le considérer comme le seul produit d'un déséquilibre entre démographie et emploi ou d'une surexploitation de la force de travail dans le cadre du capitalisme dominant, mais qu'il fallait au contraire le penser comme l'une des expressions de la réalité économique urbaine de leurs pays, par rapport à un capitalisme dominant qui n'envahit pas tous les secteurs. Et, une fois de plus, cela m'a amené à réfléchir sur les différences entre le Brésil et l'Afrique.

23 Ta façon d'aborder la question des villes du Tiers-Monde a-t-elle toujours eu le même écho chez les étudiants?

24 Je continue à penser que les phénomènes de domination sont indispensables pour comprendre les problèmes actuels des systèmes urbains, l'excentricité des grandes villes, les problèmes de désajustement de la hiérarchie urbaine, etc. Je fais toujours des cours là-dessus, et mon analyse des systèmes urbains a toujours le même écho chez les étudiants. Quand on leur explique que, pour comprendre le système urbain de ces pays, il faut revenir sur le fait qu'ils ont subi une période de domination coloniale, néocoloniale, ou semi-coloniale, et qu'on leur montre comment cela pèse encore sur les 
réalités actuelles, cela les accroche toujours. J'ai toujours de bonnes réactions de la part des étudiants auxquels je parle de ça au Brésil, en Tunisie ou en France, et en France, aussi bien devant des étudiants provenant du Tiers-Monde que devant ceux du magistère, qui sont majoritairement français - puisque dans le cadre du magistère d'aménagement et d'urbanisme j'étais le seul à parler des systèmes urbains dans le Tiers-Monde. Dans mes cours, je pars toujours de l'idée que les systèmes urbains dans le Tiers-Monde ont été, à un moment ou à un autre, modelés, non par l'évolution de la société interne, mais par un système de domination imposé par l'extérieur.

A contrario, ces idées-là, et plus globalement les problèmes du Tiers-Monde, accrochent moins dans la société française. Même la question du logement des pauvres ou celle du secteur informel, qui touchent quand même à des problèmes très directs, lorsque j'ai l'occasion d'en parler, cela n'a plus du tout le même écho. D'un côté, on s'est trop replié sur nous-mêmes et sur nos difficultés depuis vingt ans; de l'autre, le Tiers-Monde a évolué, et le côté un peu perverti des gouvernements de ces pays n'a pas aidé à en donner une bonne image. C'est ce qui explique sans doute que le grand public passe volontiers d'un intérêt émotif à une sorte de désintérêt pour le Tiers-Monde.

Tu viens de dire que les étudiants de l'Iedes venaient avec l'idée de faire quelque chose pour leur pays. Est-ce que tu sais ce que sont devenus certains de tes étudiants, est-ce que tu en as revus?

Pas beaucoup, mais j'en vois de temps en temps qui ont des postes importants. C'est le cas de quelques-uns au Brésil, et récemment, j'ai rencontré un Tunisien qui a aujourd'hui des responsabilités politiques importantes, et qui, trente ans après, se rappelle très bien de ce que l'Iedes lui a apporté comme idées qui lui ont servi de base dans son action.

Tu as dirigé beaucoup de travaux et de thèses sur le Tiers-Monde. Quelle est ta conception d'une direction de recherche? Quelle a été ton influence à ce niveau?

29 J'ai toujours pensé que diriger des recherches, c'était aider quelqu'un à accoucher de ses idées, de celles qu'il doit trouver en faisant sa thèse, et non pas lui imposer mes propres idées. J'ai toujours été très respectueux de la diversité, ce qui fait que j’ai dirigé des travaux très différents. Certains se situaient plus ou moins dans la ligne de ce que je faisais, et cela a donné des thèses effectivement intéressantes, mais d'autres n'avaient pas grand rapport avec ce que je faisais. Sur le Tiers-Monde, il y a donc des thèses que j'ai pu influencer, mais je n'ai jamais essayé de créer une "École Rochefort »; je suis complètement opposé à cela. Je n'ai jamais été un leader d'École, et même dans le financement de la recherche, puisque j'ai été responsable du financement de la recherche urbaine sur les PVD pendant six ans, j'ai surtout essayé d'aider les autres à exprimer leurs idées, et éventuellement de leur apporter le soutien financier que je pouvais leur donner. Il y a donc eu une solidarité d'approche entre certains chercheurs et moi, mais sans qu'ils soient des élèves, et j'espère ne jamais avoir travaillé avec des gens qui s'appelleraient mes élèves, au sens d'une "École Rochefort» dont les chercheurs auraient appliqué mes idées. Je veux bien qu'on dise que je les ai aidés, mais seulement cela.

30 Est-ce que le fait d'avoir travaillé sur le Tiers-Monde à partir d'un certain moment a modifié tes conceptions concernant la façon de faire de la géographie?

31 Sûrement. J'ai abordé les problèmes urbains dans le cadre de mon DES et surtout de ma thèse d'Etat sur l'organisation urbaine de l'Alsace à partir de ma formation d'historien, - puisque j'ai été historien avant d'être géographe - et j'étais très influencé par Lucien 
Febvre ce qui m'a conduit à insister sur l'impact des structures de la société dans l'organisation du territoire. À l'époque, j'étais très marqué par le fait qu'une ville, c'est la formation d'une bourgeoisie. J'abordais donc la réalité urbaine beaucoup plus au niveau des systèmes urbains que de la spécificité interne d'une ville et je m'attachais à l'analyse de la dynamique de la société, des bourgeoisies, de leur formation et de leur évolution, des faillites des bourgeoisies régionales, etc. Puis, j'ai eu une période fonctionnaliste - les métropoles d'équilibre - pendant laquelle la racine même de ma pensée - la relation entre société et espace - a été occultée. Et c'est après - plus particulièrement après 1968 - que j'ai redécouvert ça, avec le Tiers-Monde, à un moment où on parlait beaucoup d'impérialisme - peut-être un peu trop -, mais où on remettait en avant le fait que l'élément essentiel pour faire de la géographie c'était non seulement de comprendre les structures socio-économiques propres à chaque formation sociale, mais aussi les relations d'inégalités entre les sociétés. Cela m'a remis dans une géographie liée à une approche pluridisciplinaire des problèmes de territoire. Et là, je me suis davantage intéressé à des problèmes d'économie urbaine - le secteur informel ou le logement des pauvres par exemple -, sans trop les accrocher à des réalités d'organisation du territoire, mais en les rattachant plus globalement aux relations entre les groupes sociaux, et en maintenant mon questionnement sur le territoire dans une approche que j'ai voulue délibérément pluridisciplinaire. Impulser des équipes pluridisciplinaires sur les villes, c'est d'ailleurs un peu ce que j'ai essayé de faire lorsque j'étais responsable de la Section Economie/Démographie de l'Orstom, et si j'écris un dernier livre, ce sera autour de cette question. Mais, personnellement, je n'ai plus travaillé sur les pays du Nord après 1967/68. J'ai simplement dirigé des recherches et des thèses, qui étaient essentiellement orientées par le chercheur.

Le Tiers-Monde : quelle perception aujourd'hui?

32 Tu viens de nous parler de ce qui t'avait attiré à l'Iedes. Il y avait à l'époque des théories générales, voire même des doctrines, pour expliquer l'évolution du monde et la place du TiersMonde dans cette évolution globale, et ces théories générales sont actuellement complètement en repli. Dans un ouvrage paru en Scandinavie il y a quelques années, l'auteur avançait qu'une des raisons pour lesquelles on avait abandonné ces schémas généraux d'explication, c'était parce qu'ils ne tenaient pas compte des spécificités concrètes de chaque formation sociale, que s'en tenir là, c'était un peu la même chose que de vouloir s'orienter dans les bois avec la carte du monde d'un agenda de poche. Toi qui es géographe, c'est-à-dire quelqu'un qui par définition est attaché à étudier les spécificités, ou la façon dont des forces globales se concrétisent en un lieu donné, comment as-tu perçu ces théories globales et comment ta réflexion a-t-elle évolué par rapport à l'approche que l'on avait dans les années soixante-dix?

Ma réflexion reste fondée sur cette double base : celle des théories générales et de la doctrine tiers-mondiste, et celle de mon observation de géographe qui est plus attachée aux réalités d'organisation du territoire. Et ce que je continue à défendre, c'est que dans tous ces pays il y a un certain nombre d'éléments qui relèvent de phénomènes généraux, et des différences qui tiennent aux structures et aux évolutions particulières de chaque société en fonction de sa propre histoire et des périodes et formes de la domination qu'elle a subie. On peut en effet penser que le Brésil, où les phénomènes urbains sont directement liés à la domination coloniale - puisque la société brésilienne n'a pratiquement rien à voir avec l'occupation antérieure à la pénétration portugaise, les Indiens ayant été décimés -, et où la société a conquis son indépendance politique au début du XIX ${ }^{e}$ siècle, n'a pas grand rapport avec les pays d'Afrique, dont les sociétés étaient structurées avant la pénétration coloniale et où il y avait très peu de villes, mais 
où la colonisation s'est faite essentiellement pendant la seconde moitié du XIX siècle, donc avec d'autres besoins, d'autres moyens et d'autres finalités, etc. Et pourtant, quelles que soient les différences, il y a des ressemblances qui sont liées à l'impact de la domination sur la localisation des villes, à l'impact de la pauvreté sur l'espace urbain et sur le logement en particulier, et aux déséquilibres entre l'économie moderne et la population, dont le secteur informel est l'une des expressions. Partout on retrouve le secteur informel, l'habitat précaire et des systèmes urbains déséquilibrés, mais il est évident que les villes du Brésil ne sont pas celles d'Afrique, et qu'Abidjan, ce n'est pas la même chose que Calcutta.

$\mathrm{Au}$ niveau des grands ensembles, la diversité est d'abord liée aux périodes et à la structure de la domination qui s'est exercée sur ces espaces. Mais à l'intérieur de ces grands ensembles, il y a des diversités régionales, qui relèvent en partie des mêmes phénomènes. Au Brésil, il y a toutes les différences qui intéressent le géographe aux diverses échelles. Cette question a été récemment très bien discutée lors d'un séminaire sur les villes du Brésil au XVII ${ }^{\mathrm{e}}$ siècle. Le fait qu'au Brésil, dont le territoire est immense, le colonisateur se soit plus intéressé à certaines régions, parce qu'elles pouvaient produire pour l'exportation - ce qui animait l'économie de drainage -, qu'à d'autres, dont il n'avait rien à faire ou dont l'exploitation était plus ponctuelle, est à l'origine de différences au niveau des structures d'appropriation du sol et des densités de populations, qui pèsent encore sur les déséquilibres actuels. Le Nord-Est était l'essentiel de ce qui intéressait les Portugais aux XVII et XVIII ${ }^{e}$ siècles. On en revient donc toujours à l'influence de la domination, mais en recherchant cette fois - puisque finalement aucune ville ne ressemble à une autre - les différences à une autre échelle. Il y a donc à la fois ce que Yves Lacoste ${ }^{8}$ a appelé dans sa thèse Unité et diversité $d u$ TiersMonde.

35 Quand tu as rejoint l'Iedes, c'était un pôle scientifique très reconnu sur le Tiers-Monde. Lorsqu'il se passait quelque chose sur le Tiers-Monde à Paris il y avait beaucoup de chances que cela se passe à l'Iedes. Maintenant, ce n'est plus le cas. Comment analyses tu cela ?

36 J'y vois deux choses. D'abord des facteurs internes. L'Iedes a encaissé beaucoup de coups du fait d'oppositions de personnes et d'oppositions idéologiques, qui tiennent en grande partie à la position dominante, au sein de l'université de Paris I, des économistes officiels ou bien à la position du ministère, dans les moments où il était à droite. Ceux-là voulaient vraiment la mort de l'Iedes parce que cet Institut était pour eux un "ramassis de gauchistes », de « tiers-mondistes » qui compromettaient l'image de la France dans le monde. L'Iedes était donc en porte-à-faux par rapport aux forces dominantes, que ce soit au niveau de l'université de Paris I ou à certaines périodes du ministère. Et même le ministère socialiste n'a pas suivi l'Iedes.

37 Ensuite, les «tiers-mondistes" de l'Iedes étaient dans l'optique d'après 1968, des dénonciateurs du néocolonialisme, de la domination, et la société n'a plus les mêmes représentations de ces phénomènes-là. Chacun s'est un peu replié sur soi, et la crise économique n'y est pas étrangère. Donc, l'Iedes, s'il est encore un foyer de réflexion sur le Tiers-Monde, n'a plus le même écho dans la société française, parce qu'on n'y pense plus les problèmes du Tiers-Monde comme dans les années soixante-dix.

38 Après toutes ces années d'expériences sur le Tiers-Monde, qu'est-ce que tu aimerais faire aujourd'hui sur ces pays?

À la fin de ma période active, j'ai l'impression que j'ai beaucoup plus dit que je n'ai écrit. J'aime bien parler, débattre, répondre à des questions, j'aime bien que les idées 
aillent vite. Écrire c'est autre chose. Et je pense que j'ai eu des idées, mais que je ne les ai pas suffisamment approfondies, nuancées, et clairement formulées pour pouvoir les écrire, peut-être parce que j'ai trop travaillé sur des thèmes très différents. Mais j'ai aussi l'impression que dans les trois domaines du Tiers-Monde où j'ai beaucoup accompagné les recherches, je pourrais faire un livre personnalisé sur ce que j'ai pu penser sur les systèmes urbains du Tiers-Monde, sur le logement des pauvres et sur le secteur informel. J'ai fait un livre en portugais qui a été publié lors du colloque de São Paulo où sont repris mes travaux antérieurs, et cela m'a donné l'idée que, à partir de là, il pourrait y avoir un prolongement que je ferais bien en français, en faisant ressortir un petit peu plus tout ce que j'ai pu dire, et notamment ce que je pense être la « richesse » de mes cours.

41 Sur le Brésil, je suis prêt à discuter avec les Brésiliens, mais je pense que c'est à eux d'écrire sur leur pays. Le Brésil c'est un peu ma passion, c'est un pays absolument passionnant parce que très complexe. Il y a dans ce pays un fossé énorme entre une extraordinaire modernisation technique et économique et une extraordinaire permanence des relations sociales, y compris de la pauvreté. Les Brésiliens n'en sortent guère. Il y a toujours le mépris du riche pour le pauvre, la stigmatisation du pauvre qui a honte d'être pauvre, qui a des difficultés à se prendre en charge, et qui s'en remet à des leaders plus ou moins achetés par les partis politiques. La pauvreté n'est toujours pas une véritable force d'évolution sociale, et les relations sociales demeurent la plupart du temps très dures malgré la formidable modernisation technique, même s'il y a aujourd'hui des évolutions importantes avec le développement des associations de favelados par exemple. J'ai vécu cela pendant plus de quarante ans, et le Brésil n'est plus le même. Avant, on disait que c'était les villes brésiliennes et l'économie industrielle et tertiaire qui se modernisaient. Maintenant, l'agriculture a aussi beaucoup changé. Le latifundium a cédé la place aux grandes exploitations capitalistes qui gèrent des fermes de 10000 ou 15000 ha comme on gère des usines. Mais les relations de travail sont toujours très dures, et les boïas frias sont peut-être encore plus malheureux que les moradores d'avant. Il y a toujours cette dysharmonie. Dans les villes il y a une petite amélioration des favelas, mais ce n'est qu'une petite amélioration, pour diminuer le risque social, avec un maintien, voire une aggravation, des inégalités entre les riches et les pauvres, et du mépris des riches pour les pauvres qui vient des rapports maîtres/ esclaves.

Je suis tout à fait d'accord avec Gilberto Freire sur l'importance de cet héritage maître/ esclave dans le rapport entre le riche et le pauvre aujourd'hui, avec un certain racisme, contrairement à ce qu'on dit, parce que le noir est très bien quand il est pauvre, mais quand il est riche, c'est autre chose. Maintenant les Brésiliens sont contre les Japonais parce qu'ils deviennent riches, et que la vieille bourgeoisie est jalouse. Le Brésil est un pays compliqué. Il y a des archaïsmes sociaux, des inégalités sociales, qui n'ont pas été corrigés par l'extraordinaire modernisation des techniques et de l'économie industrielle et tertiaire. Il y a aussi bien des signes et des espoirs d'un avenir meilleur... 


\section{NOTES}

1. Institut d'études du développement économique et social, université de Paris I.

2.Voir dans cet ouvrage : Au Brésil : influences sur la géographie; engagement dans l'aménagement du territoire.

3.Équipe du Laboratoire de géographie humaine de Paris I associé au Cnrs, « Le rôle des activités tertiaires dans la domination des pays d'économie sous-développée ». Les travaux de cette équipe, dirigée par Michel Rochefort, ont donné lieu à la première thèse collective soutenue à Paris I en 1976. Cf. Michel Rochefort (dir), avec la collaboration de H. Lamicq, M.-C. Guerrini, C. Paix, « Les activités tertiaires supérieures dans les pays d'économie dominée », $\mathrm{n}^{\circ}$ spécial, Tiers-Monde, $\mathrm{n}^{\circ}$ 61, PUF, Paris, janvier/ mars 1975.

4.Cf. Groupe de recherche " Analyse régionale », sous la direction de Michel Rochefort, «Particularités des échanges commerciaux et différenciations spatiales en pays sousdéveloppés ; un essai de typologie des espaces dominés », IEDES, Paris, 1975, et, "Commerce extérieur et organisation de l'espace ; une typologie des espaces dominés ", Tiers-Monde, n 70, PUF, Paris, avril/juin 1977.

5.Milton Santos, L'espace partagé, les deux circuits de l'économie urbaine des pays sousdéveloppés, Éd. M. Th. Genin, Paris 1975.

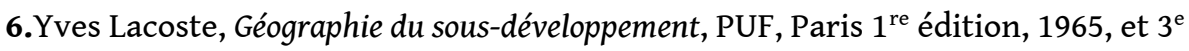
édition entièrement refondue et précédée d'un avertissement critique et autocritique, PUF, Paris, 1976.

7.Ceget : Centre de Géographie tropicale, laboratoire propre du Cnrs, Bordeaux. 8. Yves Lacoste, Unité et diversité du Tiers-Monde : des représentations planétaires aux stratégies de terrain, Paris, Éd. la Découverte, 1984.

\section{AUTEURS}

\section{MAXIME HAUBERT}

Professeur de sociologie, Iedes, université de Paris I

\section{CATHERINE PAIX}

Ladyss, Cnrs 